\title{
A NONSPECTRAL DENSE BANACH SUBALGEBRA OF THE IRRATIONAL ROTATION ALGEBRA
}

\author{
LARRY B. SCHWEITZER
}

(Communicated by Palle E. T. Jorgensen)

\begin{abstract}
We give an example of a dense, simple, unital Banach subalgebra $A$ of the irrational rotation $C^{*}$-algebra $B$, such that $A$ is not a spectral subalgebra of $B$. This answers a question posed by T. W. Palmer (Spectral algebras, Rocky Mountain J. Math. 22 (1992), 293-328).
\end{abstract}

If $A$ is a subalgebra of an algebra $B$ (both algebras over the complex numbers), we say that $A$ is a spectral subalgebra of $B$ if the quasi-invertible elements of $A$ are precisely the quasi-invertible elements of $B$ which lie in $A$. In the language of [3], this is equivalent to saying that $A$ is a spectral invariant subalgebra of $B$.

There are many known examples of dense unital Banach subalgebras of $C^{*}$ algebras which are not spectral. For example, see Example 3.1 of [3]. The example we give here is of interest because the Banach algebra is simple and, thus, answers Question 5.12 of [1] in the negative.

Recall that the irrational rotation algebra associated with an irrational real number $\theta$ is the $C^{*}$-crossed product of the integers $\mathbb{Z}$ with the commutative $C^{*}$-algebra of continuous functions on the circle $C(\mathbb{T})$, where $n \in \mathbb{Z}$ acts via $\alpha_{n}(\varphi)(z)=\varphi(z-n \theta)$, for $\varphi \in C(\mathbb{T})$ and $z \in \mathbb{T}$. Let $B=\mathbb{Z} \rtimes C(\mathbb{T})$ denote this crossed product.

Let $A$ be the set of functions $\mathrm{F}$ from $\mathbb{Z}$ to $C(\mathbb{T})$ which satisfy the integrability condition

$$
\|F\|_{A}=\sum_{n \in \mathbb{Z}} e^{|n|}\|F(n)\|_{\infty}<\infty,
$$

where \|\|$_{\infty}$ denotes the sup norm on $C(\mathbb{T})$. Then $A$ is complete for the norm \|\|$_{A}$ and is a Banach algebra. The algebra $A$ is contained in $L^{1}(\mathbb{Z}, C(\mathbb{T}))$ with dense and continuous inclusion and, hence, is contained in $B$ with dense and continuous inclusion. Recall that the multiplication (in both $A$ and $B$ ) is given by

$$
F * G(n, z)=\sum_{m \in \mathbb{Z}} F(n, z) G(n-m, z-m \theta), \quad F, G \in A, n \in \mathbb{Z}, z \in \mathbb{T} .
$$

Received by the editors June 22, 1992.

1991 Mathematics Subject Classification. Primary 46H99; Secondary 46H35, 46H25, 46L99.

Key words and phrases. Spectral subalgebra, spectral invariance, irrational rotation algebra, simple Banach algebra. 
Let $u_{n}=\delta_{n} \otimes 1 \in A$ denote the delta function at $n \in \mathbb{Z}$ tensored with the identity in $C(\mathbb{T})$. Then $u_{0}$ is the unit in both $A$ and $B$.

Theorem 1. The Banach algebra $A$ is simple.

Proof. We imitate the argument of [2]. Define a continuous linear map $P: A \rightarrow$ $C(\mathbb{T}) \subseteq A$ by $P(F)=F(0)$. Note that $\|P(F)\|_{A} \leq\|F\|_{A}$ for $F \in A$. Let $J$ be a closed two-sided ideal in $A$, which is not equal to $A$. Since $\mathbb{Z}$ acts ergodically on $\mathbb{T}$, we know that $C(\mathbb{T})$ has no nontrivial closed $\mathbb{Z}$-invariant ideals. Hence, $J \cap C(\mathbb{T})=0$.

We show that $P(J)=0$. It suffices to show that $P(J) \subseteq J$. Let $\epsilon>0$ and $F \in A$. Let $N$ be a sufficiently large integer for which

$$
\sum_{|n|>N} e^{|n|}\|F(n)\|_{\infty}<\epsilon .
$$

Define $F_{1} \in A$ by $F_{1}(n)=0$ if $|n|>N$, and $F_{1}(n)=F(n)$ if $|n| \leq N$. By the proof of Lemma 6 of [2], there exists unimodular functions $\theta_{1}, \ldots, \theta_{M} \in C(\mathbb{T})$ such that

$$
P\left(F_{1}\right)=\frac{1}{M} \sum_{n=1}^{M} \theta_{n}^{*} F_{1} \theta_{n} .
$$

(Here unimodular means that $\left|\theta_{i}(z)\right|=1$ for each $z \in \mathbb{T}$ and $i=1, \ldots, M$.) Hence,

$$
\left\|P\left(\bar{L}^{\prime}\right)-\frac{1}{M} \sum_{n=1}^{M} \theta_{n}^{*} F \theta_{n}\right\|_{A} \leq\left\|P\left(F-F_{1}\right)\right\|_{A}+\left\|F-F_{1}\right\|_{A}<2 \epsilon .
$$

Now if $F \in J,(*)$ shows that $P(F)$ can be approximated arbitrarily closely by elements of $J$. Since $J$ is closed, this shows that $P(F) \in J$. Hence, $P(J) \subseteq J$ and $P(J)=0$.

If $P\left(F u_{n}\right)=0$ for all $n$, then $F(n)=0$ for all $n$ and so $F=0$. Since $J$ is a two-sided ideal and $P(J)=0$, we have $P\left(J u_{n}\right)=0$ for all $n$. Hence, $J=0$ and $A$ is simple.

Theorem 2. The Banach algebra $A$ is not a spectral subalgebra of $B$.

Proof. We construct an algebraically irreducible $A$-module which is not contained in any *-representation of $B$ on a Hilbert space. By Corollary 1.5 of [3], it will follow that $A$ is not a spectral subalgebra of $B$.

Let $E$ be the Banach $A$-module $C(\mathbb{T})$ with sup norm and with (continuous) action of $A$ given by

$$
(F \varphi)(z)=\sum_{n} F(n, z) e^{n} \varphi(z-n \theta), \quad \varphi \in E, F \in A, z \in \mathbb{T} .
$$

We show that $E$ is in fact algebraically irreducible. Let $\varphi \in E$ be not identically equal to zero. Since the complex conjugate of $\varphi$ is in $A$, the algebraic span $A \varphi$ contains $|\varphi|^{2}$, which we denote by $\psi$. Note $u_{n} \psi(z)=e^{n} \psi(z-n \theta)$. Since $\theta$ is irrational and $\mathbb{T}$ is compact, there exists finitely many $n_{1}, \ldots, n_{k} \in \mathbb{Z}$ such that the sum of $u_{n_{i}} \psi$ from $i=1$ to $k$ never vanishes on $\mathbb{T}$. If $\chi$ is this sum, then $1 / \chi$ is in $C(\mathbb{T}) \subseteq A$, so $1 \in A \varphi$ and, hence, $E=A \varphi$. This proves that $E$ is algebraically irreducible. 
It remains to show that no *-representation of $B$ on a Hilbert space contains $E$. But the action of $\mathbb{Z}$ on $1 \in E$ is given by $u_{n} 1=e^{n} 1$. Clearly the Hilbert space could not have a unitary, or even isometric, action of $\mathbb{Z}$.

\section{REFERENCES}

1. T. W. Palmer, Spectral algebras, Rocky Mountain. J. Math. 22 (1992), 293-328. MR 93d:46079

2. S. C. Power, Simplicity of $C^{*}$-algebras of minimal dynamical systems, J. London Math. Soc. (2) 18 (1978), 534-538. MR 81e:46057

3. L. B. Schweitzer, $A$ short proof that $M_{n}(A)$ is local if $A$ is local and Fréchet, Internat. J. Math. 3 (1992), 581-589. MR 93i:46082

Department of Mathematics, University of Calgary, Alberta, Canada T2N 1N4

Department of Mathematics, University of California, Berkeley, California 94720

Current address: Department of Mathematics and Statistics, University of Victoria, Victoria, British Columbia, Canada V8W 3P4

E-mail address: 1schweit@alpha.math.uvic.ca 